\title{
La emergencia de redes clientelares en América Latina: Una perspectiva teórica
}

\author{
The Emergence of Clientelistic Networks in Latin America: A Theoretical Perspective
}

\section{Carlos Hoevel}

Centro de Estudios en Economía y Cultura, Facultad de Ciencias Económicas, Pontifica Universidad Católica Argentina, Argentina

\author{
Aldo Mascareño \\ Centro de Investigación Núcleo Milenio Modelos de Crisis, Escuela de Gobierno, Universidad Adolfo \\ Ibáñez, Chile
}

\begin{abstract}
RESUMEN
Las redes clientelares han sido objeto de investigación recurrente en América Latina particularmente en las últimas tres décadas. Esta investigación ha enfatizado en sus modalidades de funcionamiento y consecuencias para el contexto democrático. No obstante, menos atención se ha puesto en perfilar las condiciones de emergencia de las redes clientelares en América Latina y la contribución que la teoría sociológica contemporánea puede hacer para ampliar el espectro analítico del campo. Por medio del recurso a distintas teorías sociológicas actuales (especialmente teoría de sistemas y sociología relacional) y a fuentes de investigación empírica sobre redes clientelares, en este artículo argumentamos que ellas emergen en América Latina en un contexto operativo de crisis de modernización que afecta fuertemente el modus vivendi personal. Esta crisis produce un contexto simbólico de deflación de compromisos valóricos que las redes clientelares solo pueden enfrentar con efectos relacionales limitados, los que, adicionalmente, impactan negativamente en la generalización de la confianza social.
\end{abstract}

PALABRAS Clave: Redes clientelares; Crisis de modernización; Sistemas sociales; Modus vivendi; Compromisos valóricos; Relaciones sociales; Confianza

\section{ABSTRACT}

Clientelistic networks have been a regular research topic in Latin American scholarship, particularly over the last three decades. This research has comprehensively addressed their functioning and consequences for democracy. However, less attention has been paid to draw the conditions of emergence of clientelistic networks in Latin America and the contribution contemporary sociological theory can make to widen the analytical scope of the field. By elaborating on different general sociological theories (particularly systems theory and relational sociology) and empirical research on the topic, this article argues that clientelistic networks in Latin America emerge in an operative context of modernization crisis that strongly disturbs the personal modus vivendi. This crisis produces a symbolic framework of deflating value commitments clientelistic networks can deal with only with limited relational effects, that, additionally, negatively impact on the generalization of social trust.

KEYWORDS: Clientelistic networks; Modernization crisis; Social systems; Modus vivendi; Value commitments; Social relations; Trust

\footnotetext{
REVISTA MAD - REVISTA DEL MAGÍSTER EN ANÁLISIS SISTÉMICO APLICADO A LA SOCIEDAD ISSN 0718-0527

Facultad de Ciencias Sociales, Departamento de Antropología.

Universidad de Chile

www.revistamad.uchile.cl
}

DOI: http://dx.doi.org/10.5354/0718-0527.2016.40612 


\section{INTRODUCCIÓN}

América Latina parece manifestar en su dinámica social la persistencia de una suerte de rutina consistente en un estado casi permanente de oscilación entre la tendencia a la imposición institucional modernizadora y la resistencia a esta imposición. A diferencia de los países europeos, cuya identidad fue formada mucho antes del inicio de esta dialéctica típica del proceso de modernización, y de los Estados Unidos, cuyas instituciones emergieron gradualmente antes de su constitución como nación independiente de un modo más bien autónomo en relación a la metrópoli europea, en América Latina tanto la estrategia de imposición forzada de instituciones por parte de España -con escasa participación y posibilidades de experimentación de la población local- y la consiguiente estrategia de respuesta por medio del falso cumplimiento o de abierta resistencia al orden institucional por parte de la población, arraigaron fuertemente en el núcleo mismo de la dinámica social nacional en América Latina (Germani 1962, 1981; Larraín 2000; Araujo 2009; Girola 2011).

Si bien la transferencia de instituciones modernas desde países centrales tuvo éxito en el objetivo de formación de Estados nacionales hacia fines del siglo XIX, el proceso general tuvo un carácter elitista de "modernización inducida". Esto significa que elites comerciales e ilustradas, para conducir el proceso modernizador, hacen uso de categorías de pensamiento y acción que no se ajustan al nivel de complejidad de su objeto -en este caso de las relaciones sociales en América Latina: el problema "[no] es enfocado en su gravedad social y realidad histórica, sino tematizado ideológicamente a partir de las tradiciones epistemológicas de los centros" (Chávez \& Mujica 2015: 77). La conformación elitista de la sociedad latinoamericana y su carácter excluyente entró en contradicción con el principio de igualdad y universalidad que impulsa las instituciones modernas (Poggi 1978; Chernilo 2007). Especialmente la formación de una creciente clase obrera, el desarrollo de una clase media con progresivas aspiraciones de inclusión, y las exigencias de reconocimiento de identidades indígenas y de diversidad cultural, incrementaron y multiplicaron las demandas hacia un Estado que no tenía las capacidades de inclusión requeridas (Domingues 2008; Bringel y Domingues 2015). A partir de ahí surgió en toda su fuerza la dialéctica de modernización entre un Estado elitista y amplios grupos 
excluidos que desconfían de las instituciones modernas, precisamente por su incapacidad de cumplir sus compromisos.

En la literatura sobre procesos de modernización existe consenso en relación a que una de las formas que adopta esta resistencia son las redes clientelares o de patronazgo (Eisenstadt \& Roniger 1984; Torsello 2012). Las redes clientelares, basadas en un principio de reciprocidad de favores y no de derechos universales, se pueden caracterizar como un "sistema de transferencias mutuas": el patrón (político, empresario, sindicalista, sacerdote, profesor) pone a disposición de sus seguidores protección y determinados rendimientos sociales (acceso, información, servicios, disponibilidad de objetos), mientras que los seguidores responden con contraprestaciones (fidelidad, trabajo, votos) (Aguirre 2012; Narotzky \& Moreno 2002). Además de este componente transaccional, las redes clientelares forman un vínculo de confianza interpersonal limitado únicamente a los miembros del grupo -generalmente familiares, amigos, seguidores o fieles al patrón-y consecuentemente extiende una actitud de desconfianza e incluso de hostilidad o violencia potencial hacia todo el resto de las personas que están fuera del círculo clientelar (Gambetta 2009).

En los últimos treinta años, la investigación socio-antropológica en América Latina y en otras regiones de la sociedad mundial ha descrito en detalle los modos de funcionamiento de las redes clientelares, así como sus consecuencias para las instituciones democráticas (Lomnitz 1988; Durston 1999; Auyero 2000, 2012; Escobar 2002; Helmke \& Levitsky 2006; Kitschelt \& Wilkinson 2007). Esta investigación no ha estado exenta de reflexiones de teoría sociológica general, aun cuando ello no ha sido su foco. Concordamos con Magdalena Tosoni (2010) en la necesidad de emplear la teoría sociológica para situar el análisis del fenómeno en un contexto más amplio de relaciones sociales. No obstante, agregamos a esto la necesidad de profundizar teóricamente no solo en el esquema que sostiene y reproduce las redes clientelares, sino en sus "condiciones de emergencia", es decir, en el mecanismo que permite observar el surgimiento de las propiedades autónomas del fenómeno a explicar (Maturana 2009; Archer 2009).

En este artículo buscamos identificar el mecanismo de emergencia de las redes clientelares modernas en América Latina. Nuestro argumento sostiene que, lejos de ser una forma de supervivencia del pasado que un proyecto político de radicalización de las instituciones modernas podría 
extirpar, las redes clientelares constituyen un producto del propio proceso de modernización en un doble sentido.

En primer lugar, desde un punto de vista 'operativo', ellas sostienen una lógica de resistencia frente a la exclusión histórica producida por una modernización inducida de sustrato elitista que genera expectativas que exceden la capacidad de implementación efectiva de las instituciones sociales en América Latina. Es decir, modernización y redes clientelares son co-originarias, en el sentido de que emergen paralelamente en una relación de complementariedad: la primera, en términos de un complejo sistémico-institucional de mecanismos y procedimientos principalmente asociados al proceso de diferenciación funcional; las segundas, en términos de vínculos de confianza interpersonal limitados a miembros de un grupo particular que busca suplir los vacíos del proceso de modernización y se mezclan con él, buscando preservar u obtener privilegios de inclusión. Esta diferencia entre expectativas y capacidades sistémico-institucionales constituye la forma de una "crisis de modernización" expresada en un sostenido descenso de la confianza institucional. Frente a esto, las redes clientelares emergen como un mecanismo alternativo para la implementación de los planes de vida cuando, producto de la crisis, las condiciones sistémico-institucionales se vuelven extremas.

En segundo lugar, desde un punto de vista 'simbólico', los problemas de generación de confianza institucional socavan el modelo reflexivo de vínculo social moderno fundado en la igualdad de individuos y la universalidad de derechos. Esto conduce a una deflación de compromisos valóricos que limita la capacidad asociativa y relacional de la sociedad en diversos ámbitos. Si bien las redes clientelares pueden producir vínculos relacionales, el carácter privatista y fragmentario de ellas opera de modo contrario a la recomposición de una confianza social generalizada.

Para desarrollar este argumento, la primera sección el artículo busca perfilar conceptualmente las condiciones de emergencia de las redes clientelares en América Latina, con apoyo en la teoría sociológica sobre este tema y en algunas evidencias empíricas. Con ello buscamos distinguir el fenómeno de las redes clientelares de otros tipos de redes sociales (redes asociativas) y a la vez desarrollar un conjunto de proposiciones adecuadas al esclarecimiento del fenómeno. Formulamos dos proposiciones para referir al 'contexto operativo' de emergencia de las redes clientelares en América Latina, y dos para referir al 'contexto 
simbólico'. En la sección 2 nos hacemos cargo de las dos primeras proposiciones. Elaboramos aquí una explicación de lo que entendemos por crisis de modernización y las consecuencias que ella tiene para la realización de planes personales de vida y la emergencia de redes clientelares. En la sección 3 abordamos las dos últimas proposiciones. Relacionamos aquí la deflación de compromisos valóricos producida por la crisis de modernización con la emergencia de redes clientelares y evaluamos sus consecuencias desde una perspectiva relacional. Finalizamos el artículo con una conclusión que sintetiza los principales hallazgos, limitaciones y líneas de investigación futura.

1. Redes CLIENTELARES EN AMÉRICA LATINA: UNA HIPÓTESIS DE SU EMERGENCIA

Desde nuestra perspectiva, la emergencia de redes clientelares en América Latina es consecuencia de una "crisis de modernización". Este tipo de crisis emerge cuando a nivel sistémico existen problemas regulatorios no resueltos que afectan la integración social. Estos problemas regulatorios tienen su origen en la relación co-originaria de diferenciación institucional y vínculos clientelares orientados a la preservación o mantención de privilegios de inclusión social. Esto produce efectos no esperados en las expectativas del "sistema de instituciones en el cual los sujetos hablantes y actuantes se encuentran socialmente relacionados" (Habermas 1973: 4). Las instituciones modernas prometen una inclusión que las redes clientelares co-originarias limitan. La crisis, por tanto, tiene una doble dimensión. Por un lado aparecen problemas regulatorios sistémicos tales como una limitada capacidad de inclusión que divide a la población en zonas de inclusión y de exclusión (Luhmann 2005, 2007), debilidad en las barreras de transferencia entre sistemas que produce una avalancha de exclusiones (Stichweh 2005), aceleración de los flujos de convertibilidad entre rendimientos sistémicos que acrecientan la diferencia entre igualdad y desigualdad (Cadenas 2012a, 2016), asincronía entre operaciones de distintos sistemas que se refleja en dificultades de coordinación de rendimientos y servicios y en problemas de desdiferenciación (Mascareño 2012), e incapacidad sistémica de hacerse cargo de sus propias externalidades producto de su tendencia inmanente a reproducir la propia lógica funcional (Teubner 2012). Por otro lado, la crisis se experimenta desde la perspectiva de las personas como un obstáculo muchas veces insalvable para la concretización del modus vivendi individual y del círculo 
de intimidad (Archer 2007). El "modus vivendi" es una constelación de intereses, necesidades y aspiraciones que los individuos priorizan de acuerdo con las constricciones y posibilidades que encuentran en su relación con estructuras sociales. Éste "rara vez es óptimo, es frecuentemente revisable, pero es siempre reflexivo por naturaleza" (Archer 2007: 88). La experiencia de crisis exige al máximo las capacidades reflexivas del "modus vivendi" y puede conducir a las personas a privilegiar opciones que en otras condiciones no seleccionarían.

En tal sentido, nuestra Proposición 1 es que: 'El contexto de emergencia de las redes clientelares en América Latina se caracteriza por una crisis de modernización en la que problemas sistémicos regulatorios constriñen fuertemente los modus vivendi de las personas, quienes privilegian alternativas extra-institucionales para realizar sus planes de vida'.

Específicamente, este contexto de emergencia de las redes clientelares se expresa en América Latina en dos niveles:

a) Una alta desigualdad en el acceso a los rendimientos de sistemas sociales que produce diversas formas de exclusión social, y

b) Una declinación de la confianza sistémico-institucional especialmente en instituciones políticas y económicas encargadas de realizar compromisos de inclusión.

Si bien los indicadores de pobreza han descendido en la región desde la década de 1980 en adelante $-48,4 \%$ en 1990; 43,8\% en 1999; 28,1\% en 2012 (Cepal 2015) - y los coeficientes Gini muestran mejorías relativas en la distribución de ingresos -reducción del índice de 0,542 en 2002 a 0,486 en 2013 (Cepal 2014)-, la desigualdad en el acceso a rendimientos de sistemas sociales sigue mostrando disparidades entre segmentos de la población (diferenciados por género, raza, etnia, o períodos de vida como infancia, juventud, vejez) y por zonas geográficas al interior de los países entre zonas urbanas y rurales (Cepal 2015) o incluso en los propios centros urbanos entre diversos segmentos de población (Heinrichs 2011). Esta situación de disparidad en el acceso a rendimientos de sistemas sociales impacta fuertemente en las posibilidades de realización del modus vivendi y conduce a un persistente descenso en los niveles de confianza institucional en América Latina. Según datos de Latinobarómetro (2015), particularmente en las instituciones de la democracia (partidos, congreso, poder judicial, gobierno), el nivel de confianza no supera los 4 de 10 latinoamericanos entre 1995 y 2015; las empresas privadas muestran un ciclo más asociado 
a las oscilaciones económicas: bajan de un 45\% sostenido entre 2005 y 2010 a 40\% desde 2011 a 2015; una situación similar a la que enfrentan los medios de comunicación, que descienden bajo el 50\% desde 2008 en adelante.

De interés para nuestro argumento es también la composición de la confianza interpersonal en América Latina. El mayor nivel de confianza interpersonal se establece con colegas de trabajo (70\%) y con vecinos (63\%); los extranjeros (28\%) y telefonistas de centros de llamados (22\%) muestran las menores cifras (Latinobarómetro 2015). Ello indica que la confianza interpersonal puede exceder los límites familiares y no estar determinada religiosamente, como algunas investigaciones sobre América Latina lo han sostenido (Parker 1990; Cousiño \& Valenzuela 1996). Más importante aún, la esfera funcional del trabajo es un espacio para el desarrollo de relaciones de confianza interpersonal. Si bien los mercados, las empresas y la política se pueden ver como sistemas que operan de acuerdo con la lógica de diferenciación funcional (Luhmann 2007), también son espacios relacionales donde los individuos se reconocen mutuamente como personas (Archer 2007, 2013; Donati 2011; Donati \& Archer 2015). Este proceso de reconocimiento mutuo no es un complemento extrínseco a los sistemas económicos o políticos, sino que es fundamental para su buen funcionamiento (Holzer 2006). Así, es posible pensar los sistemas sociales también "como sistemas de interdependencia, caracterizados por una creciente interacción y relacionalidad" (Donati 2010: 62).

De este modo, puede experimentarse baja confianza institucional en la empresa, pero la afirmación de lazos personales con colegas de trabajo opera como sustituto de la desconfianza en el sistema. Bajo estos términos, nuestra Proposición 2 es que: 'Los vínculos personales que caracterizan a las redes clientelares abren posibilidades reflexivas de realización del modus vivendi en espacios funcionales con disparidad en el acceso a rendimientos de sistemas sociales'.

Cuando las relaciones sociales se establecen de manera asociativa y descentralizada, se incrementa la probabilidad de desarrollar un marco sistémico con capacidad de procesamiento de las demandas de los públicos. Si esto tiene lugar, aumenta la confianza institucional, como en particular acontece en países escandinavos con cifras de confianza entre $60 \%$ y $70 \%$, las que duplican a países del sur europeo como Portugal, Grecia o Italia (Albanese \& De Blasio 2014). En aquellos casos, los rendimientos sistémicos de instituciones políticas y económicas logran 
sustentar la aspiración normativa de igualdad de individuos y universalidad de derechos. Esto establece un piso de relativa seguridad sobre el cual se pueden formar vínculos interpersonales de tipo horizontal, asociativo y descentralizado (Rothstein \& Stolle 2003): se puede confiar en otro lejano o incluso desconocido cuando un nivel relativamente alto de confianza sistémico-institucional provee de una base para tomar esos riesgos (Luhmann 2000).

Desde nuestra perspectiva, esta situación constituye una forma de modernización que integra la dimensión sistémica y la relacional, y que incluso puede canalizar sus conflictos por medio de un incremento de esa integración a través de consejos sociales, sistemas deliberativos o de negociación y redes de actores que coordinan descentralizadamente posiciones e intereses diferenciados (Willke 2014). Sin embargo, cuando el contexto sistémico-institucional es incapaz de implementar sus compromisos de inclusión, como acontece en América Latina, entonces la irrealizada aspiración normativa de igualdad de individuos y universalidad de derechos se revierte en términos de discriminación institucionalizada, que produce problemas de diferenciación y legitimación del derecho (Cadenas 2012b) y una baja confianza institucional o deflación de los compromisos valóricos generalizados (Parsons 1968). En esta situación deflacionaria no existe base social vinculante para generar formas de confianza de manera asociativa y descentralizada. La alternativa de vínculo se busca en espacios de familiaridad o en redes de tipo vertical que permitan una conexión con actores situados en posiciones de privilegio en contextos organizacionales de distinto tipo, públicos o privados, con los cuales se pueda establecer un sistema de transferencias mutuas de tipo clientelar (Putnam 1993). La consecuencia de ello es que el espacio social se comunitariza y fragmenta.

De esta forma, nuestra Proposición 3 es que: 'Las redes clientelares emergen en América Latina en una situación social de deflación de los compromisos valóricos de inclusión igualitaria y universalista, lo que promueve modalidades de acción particularizada basadas en favores propias del mecanismo clientelar de transferencias mutuas'.

Puesto en términos sintéticos, el mecanismo de emergencia de redes clientelares modernas que proponemos para América Latina es el siguiente. Por un lado, un contexto sistémico-institucional históricamente elitista no logra absorber de manera igualitaria y universal las demandas públicas, y por otro, este mismo sesgo excluyente dificulta la formación de redes asociativas horizontales que se integren de modo relacional con la 
lógica funcional. Como respuesta a esta situación de exclusión y de deflación de compromisos valóricos de inclusión igualitaria y universalista, las exigencias sociales de relacionabilidad se canalizan -no exclusivamente, pero de manera importante- por la vía de vínculos de red que reproducen, en distintos niveles, un modelo clientelar. En este sentido, las redes clientelares emergen co-originariamente con el modelo sistémico-institucional moderno, constituyendo una alternativa de inclusión frente a él, pero también tensionándolo de manera importante hacia la preservación o producción de privilegios para grupos específicos.

Este modelo abre un esquema conflictual constitutivo. La red clientelar puede suplir en parte el vacío de asociatividad, pero a la vez promueve pequeñas redes de reciprocidad fragmentada y conflictivas entre sí o, al menos, inmersas en un clima general de desconfianza mutua (Hoevel 2014). El fenómeno se complejiza aún más con la penetración de estas redes en la estructura institucional del Estado. Ello produce una cooptación del espacio público como espacio clientelar, caracterizado por ámbitos en los que la imposición legítima del derecho se suspende (Oliveros 2013). La formación de este espacio clientelar que sustituye relaciones basadas en una confianza social extendida y establece un espacio normativo privatizado y ambiguo, sirve a las partes en el corto plazo, pero afecta la producción de bienes relacionales en el largo plazo. Como lo ha hecho ver Robert Putnam (1993), en base a un estudio comparativo entre el norte y el sur de Italia, el efecto general de estas redes clientelares sobre las instituciones sociales es desconfianza, fragmentación, dependencia vertical, explotación, criminalidad y desfases en bienestar general. Un argumento similar fue desarrollado por Diego Gambetta (1990), quien, en sus análisis sobre la estructura social del sur italiano, ha enfatizando los problemas de las redes clientelares, las cuales, llevadas a su último extremo, conducen a la conformación de las sociedades mafiosas.

En este sentido, nuestra Proposición 4 y última es que: 'Las redes clientelares forman un espacio social de tipo transaccional con producción de bienes relacionales, pero con consecuencias negativas para la generalización social de esos bienes'. 
2. CRISIS SISTÉMICA Y CONSTRICCIÓN DEL MODUS VIVENDI: EL CONTEXTO OPERATIVO DE LA EMERGENCIA DE REDES CLIENTELARES

En esta sección buscamos fundar teóricamente las dos primeras proposiciones de nuestro enfoque. Esto es, (1) que 'el contexto de emergencia de las redes clientelares en América Latina se caracteriza por una crisis de modernización en la que problemas sistémicos regulatorios constriñen fuertemente los modus vivendi de las personas, quienes privilegian alternativas extra-institucionales para realizar sus planes de vida' y (2) que 'los vínculos personales que caracterizan a las redes clientelares abren posibilidades reflexivas de realización del modus vivendi en espacios funcionales con disparidad en el acceso a rendimientos de sistemas sociales'.

Puesto que el objetivo de este artículo es investigar las condiciones de emergencia de las redes clientelares y sustentarlas en teoría sociológica, en esta sección nos enfocamos en el 'contexto operativo' y dejamos para la siguiente sección el 'contexto simbólico' y la fundamentación de las dos últimas proposiciones hechas en la primera sección. En tanto nuestro foco es América Latina, incorporamos literatura regional sobre el tema en ambas secciones y hacemos también mención a investigaciones en otras regiones de la sociedad mundial.

A nuestro entender, la sociedad latinoamericana experimenta actualmente una crisis de modernización que ha sido interpretada de distintos modos. Gino Germani (1981), en el marco de las teorías de la modernización, observó esta crisis por medio de desarrollos asincrónicos entre componentes institucionales, grupales, motivacionales y geográficos de la vida social. Pedro Morandé (1987) la interpretó como una imposición alienante de la racionalidad instrumental sobre un sustrato cultural católico-indígena latinoamericano. Norbert Lechner (1999), en base a una lectura integrada de Jürgen Habermas y Niklas Luhmann, pone de relieve una desconexión entre modernización y subjetividad, en tanto el despliegue de la lógica de mercado en América Latina niega las posibilidades de intervención subjetiva en el marco funcional. Manuel A. Garretón (2000) ha leído esta crisis desde un punto de vista fundamentalmente político como una desestabilización de lo que denomina "matriz sociopolítica", compuesta por Estado, partidos y sociedad civil. Jorge Larraín (2000) ha revisado el problema como un permanente desajuste entre proyectos modernizadores y desarrollos identitarios. Walter Mignolo (2005), en el marco de teorías decoloniales, 
ha llamado la atención sobre el esquema de dominación epistemológica fundamental de 'América Latina' como sociedad postcolonial. Y José M. Domingues (2008) ha situado la crisis en la contradicción entre un desarrollo democrático amplio del continente expresado en diversidad de movimientos emancipatorios y el carácter inequitativo del proyecto neoliberal.

En nuestra interpretación, la crisis de modernización en América Latina es una crisis de complejidad sistémica que se produce por el crecimiento compulsivo (Teubner 2012) de racionalidades sistémicas, particularmente de la política (politización de la sociedad) y de la economía (monetarización de la sociedad). Al contrario de la interpretación común que observa en los sistemas funcionales una tendencia hacia la estabilidad y la morfoestásis (Habermas 1973; Archer 2013), el imperativo sistémico es la 'conectividad' de la comunicación, esto es, la tendencia inmanente de los sistemas sociales a reproducir su propia lógica en todos sus eventos comunicativos. Esta predisposición provoca una alta inestabilidad en los sistemas y conduce a un proceso de redundancia comunicativa que denominamos "lógica del exceso" (Mascareño, Goles, \& Ruz 2016) -ella puede ser observada de manera clara en la crisis financiera 2008, en la que el sistema financiero continua con la producción de derivativos, aún después de superar sus condiciones de demanda (Haldane \& May 2011). Esta lógica del exceso genera fenómenos de desborde comunicativo (Callon 1998) que sobrepasan las barreras de contención de influencias sistémicas mutuas (Stichweh 2005) e intervienen y desdiferencian otras lógicas autónomas de sistemas sociales. En el caso latinoamericano de la última mitad del siglo XX e inicios del XXI, esto se expresa en procesos de politización de la sociedad, como en el caso del populismo, o de monetarización, como en el caso del neoliberalismo (Mascareño 2012), situaciones sociales en las que respectivamente las lógicas sistémicas de la política y la economía intervienen otros espacios sociales para reproducir su propia complejidad.

De manera adicional a lo anterior, estos procesos de desborde y desdiferenciación se ven acrecentados por intereses particulares, tanto de élites como también -especialmente en las últimas décadas del siglo XX e inicios del XXI- de estratos medios y bajos, que prevalecen sobre el interés general y que manejan el proceso de diferenciación hacia la consecución de sus objetivos a través de redes clientelares. El modelo sistémicoinstitucional se expande $y$ al mismo tiempo es interpenetrado parasitariamente por estas redes, cuyos objetivos no son solo la inclusión, 
sino también la defensa de una cierta "actitud preventiva" frente a las tendencias homogenizadoras de la diferenciación de sistemas que limita el espacio operativo y simbólico para la expresión de diversos modus vivendi (Hoevel 2014).

Cuando esto acontece, la relación co-originaria de diferenciación sistémica y redes clientelares actúa unívocamente. Los intereses particulares de grupos específicos cooptan marcos institucionales determinados y los llevan a operar en beneficio propio. De ese modo, el propio proceso de diferenciación adopta un carácter de crecimiento compulsivo y de excesos originados en la reproducción de la conectividad sistémica dirigida hacia una finalidad específica de grupos particulares, sean de estratos altos (colusiones de mercado), medios (cadenas de favores en administración pública) o bajos (redes de comercio informal o microtráfico). El proceso de modernización en general se vuelve entonces más incontrolable desde el sistema político, o más auto-incontrolable para los propios mecanismos de auto-regulación sistémica. Se producen procesos de "hipertrofia sistémica" (Neves 2007), cuya consecuencia 'operativa' es la incapacidad generalizada de las instituciones sociales de absorber y procesar las crecientes demandas de los públicos; y cuya consecuencia 'simbólica' es la imposibilidad de cumplir la expectativa normativa de igualdad de individuos y universalidad de derechos -con la consiguiente pérdida de confianza institucional, como lo hemos sostenido en la primera sección.

No es por tanto solo el Estado el que está en crisis, sino el proceso de modernización en general, en cuanto está basado en sistemas funcionales con bajos índices de confianza producto de la propia tendencia autoinmunitaria de los sistemas de ponerse en peligro a sí mismos. La diferenciación funcional tiene el problema de la pérdida gradual de una racionalidad general. Si bien los sistemas funcionales poseen mecanismos de producción y autorregulación, estos se basan siempre en medios propios que solo observan los problemas de las personas en tanto esos sistemas se vean afectados. Como el propio Luhmann lo expresa en un tono altamente pesimista hacia el final de su vida:

Hoy el problema es mucho peor que antes. Podemos continuar con nuestros hábitos y volver a demandas morales que estarán tan justificadas como siempre, pero ¿quién escuchará esas quejas y quién reaccionará ante ellas si la sociedad no puede controlarse a sí misma? ¿Y qué podemos esperar si sabemos que el mismo éxito de los sistemas funcionales depende de su indiferencia? Cuando la evolución ha diferenciado sistemas cuya complejidad depende de la 
clausura operacional [...] ¿cómo podemos esperar incluir todo tipo de preocupaciones dentro del sistema? (Luhmann 1997: 74s)

En la medida en que constituyen una forma de acceso a los rendimientos de los sistemas sociales, cuando el contexto institucional no produce un cumplimiento efectivo de sus compromisos de inclusión igualitaria y universal, las redes clientelares son una respuesta a la pregunta de Luhmann. Las situaciones de crisis social afectan el funcionamiento de sistemas sociales, pero más importante que ello es que afectan los planes de vida de las personas y sus círculos íntimos. Como lo ha sostenido Habermas (1973: 1): "La crisis no puede ser separada del punto de vista de quien atraviesa por ella [...] Por tanto, asociamos con crisis la idea de una fuerza objetiva que depriva al sujeto de alguna parte de su normal soberanía". En nuestra interpretación, aquella 'fuerza objetiva' es la indiferencia sistémica transformada en crisis por la lógica del exceso que integra diferenciación institucional con clientelismo. Y la deprivación que produce es una constricción fuerte en el modus vivendi personal.

Para Margaret Archer (2007: 62ss, 2000: 222ss), el "modus vivendi" refiere a una constelación de preocupaciones (concerns) que los individuos construyen a lo largo de su experiencia en y con la sociedad, y que esperan sea satisfactorio y sustentable, en tanto comprenda "un conjunto establecido de prácticas que constituyan una forma de vida deseable definida por el sujeto a la luz de sus preocupaciones últimas" (Archer 2007: 223). Pierpaolo Donati (2015a: 91) lo ha definido de manera similar: "[El modus vivendi es] una forma de conducir la existencia con otros en una esfera social no diferenciada de acuerdo a funciones, sino a relaciones. Es decir, se distingue por sus cualidades sui generis y los poderes que lo constituyen". La experiencia relacional con otros y con estructuras sociales lleva a los individuos a priorizar sus preocupaciones (intereses, necesidades, aspiraciones, imágenes de sí mismo, en una palabra: expectativas) atendiendo a las constricciones y habilitaciones que ellos encuentran en distintas situaciones sociales. El modus vivendi es por tanto una guía normativa y cognitiva de los individuos para orientar sus cursos de acción social. Sin embargo, elegir un curso de acción en una determinada situación social supone reflexividad individual para decidir qué tipo de motivación puede ser puesta en práctica. Ello exige un contexto institucional que ofrezca un conjunto relativamente amplio de opciones para que la elección reflexiva del curso de acción pueda tener 
efectivamente lugar. De este modo, sostenemos que cuando las condiciones institucionales se caracterizan por desigualdad, desconfianza, elitismo, incapacidad de procesar demandas múltiples y disparidades de acceso, todos elementos que conducen a una situación de crisis de modernización, como hemos observado acontece en el caso latinoamericano, entonces el modus vivendi se ve fuertemente constreñido a alternativas limitadas.

Sin embargo, puesto que el modus vivendi tiene un componente normativo, no puede renunciar a sus preocupaciones fundamentales, aun cuando la situación social que se enfrente sea de 'crisis objetiva'. Pero puesto que también tiene un componente cognitivo reflexivo, los individuos pueden implementar su actitud preventiva y crear alternativas relacionales, con el fin acceder a recursos que contribuyan a implementar sus planes de vida cuando el contexto institucional no otorga alternativas de acceso o cuando los presiona hacia una homogenización social. La emergencia de las redes clientelares en el contexto latinoamericano tiene justamente esta génesis. Particularmente las investigaciones de Javier Auyero $(1999,2010,2012)$ han demostrado que las redes clientelares se entienden solo parcialmente cuando se las ve como un mecanismo de dominación política top-down. Parte importante de la motivación para su emergencia proviene de la búsqueda de alternativas de acceso en condiciones sistémicas extremas:

En vecindarios pobres y de clase trabajadora, barriadas (shanty-towns) y tomas ilegales a lo largo de todo el país [Argentina], muchos de los pobres y desempleados solucionan sus acuciantes problemas cotidianos (acceso a comida y medicina, por ejemplo) a través de redes de patronazgo que descansan en brokers del partido peronista (localmente conocidos como punteros) como actores clave. (Auyero 2012: 100)

Distintas investigaciones en las favelas brasileñas han constatado situaciones similares. Veronika Deffner (2007: 215), por ejemplo, llama la atención sobre las condiciones de seguridad y protección que ofrecen las redes clientelares con carteles de droga a los habitantes ante "la ausencia de poder institucional y el desplome de la policía y el orden público". Enrique Arias y Corinne Rodrigues (2006) muestran cómo los habitantes obtienen abastecimiento de agua, financiamiento, acceso mercancías robadas e incluso transporte a centros comerciales y hospitales fuera de las favelas por parte de las organizaciones criminales. Clarissa Huguet \& Ilona de Carvalho (2008) enfatizan en la dependencia de algunos grupos de habitantes de las favelas de los servicios generalmente ilegales que 
ofrecen las redes clientelares con organizaciones criminales. Y Gabriel Feltran (2010) hace evidente cómo en zonas periféricas de São Paulo los "tribunales del crimen" desarrollan una justicia paralela a la justicia estatal basada en códigos criminales y a la cual los pobladores acuden en tanto los tribunales ordinarios les son inaccesibles. La constante es lograr un modus vivendi en condiciones sociales extremas.

Fue justamente el caso brasileño el que motivó a Luhmann a abordar el tema de las redes clientelares en el marco de la distinción inclusión/exclusión. Para Luhmann (2005), las redes clientelares surgen típicamente en países en vías de modernización, donde el proceso de diferenciación funcional no logra circunscribir desigualdades a ámbitos funcionales específicos y donde es incapaz además de modificar tales desigualdades rápidamente. En esta situación social se produce una disociación entre ámbitos de inclusión y ámbitos de exclusión, en los que "gran parte de la población, de manera estable, queda excluida de la participación de los beneficios funcionales" (Luhmann 2005: 235). Las redes clientelares actúan como mecanismos de reconexión entre los ámbitos de inclusión y exclusión por medio del acceso a posiciones funcionales y organizacionales que se emplean de modo parasitario, provocando cortocircuitos y corrompiendo la racionalidad funcional (Luhmann 2005). Bajo esta modalidad de diferenciación funcional, se puede actuar legalmente (con interés universal) e ilegalmente (con interés particular) desde las posiciones ocupadas en las organizaciones. El mecanismo que Luhmann identifica es el siguiente:

El recurso básico de esta red parece ser que alguien conoce a alguien que conoce a alguien; y que la solicitud de favores está de tal modo extendida que cuando se tiene la posibilidad de ayudar, esta no se puede rechazar sin quedar dentro de corto tiempo excluido de la red de servicios recíprocos. La red de servicios recíprocos genera su propio mecanismo de exclusión: puede llegar a causar que alguien se constituya en no-persona en la medida que, a pesar de haber cumplido todas las formalidades requeridas, por no tener quien lo conozca, no pueda tener acceso a los sistemas de funciones. La estructura del contacto se basa típicamente en la interacción face to face [...] y facilita (en términos parsonianos) una orientación de temas particular pero no universalista, difusa pero no específica. En esta interacción se trata en todo caso de inclusión de la persona en su totalidad. (Luhmann 2005: 236)

Varios elementos de este análisis complementan nuestra aproximación. En primer lugar, Luhmann sostiene aquí la tesis transaccional y relacional de un sistema de transferencias mutuas para las 
redes clientelares, también argumentada en la literatura empírica. No se trata meramente de un intercambio funcional, sino de un esquema interaccional en el que son las personas las que individualmente importan: para la solicitud de favores se requiere 'conocer a alguien' y no solo tratarlo como 'portador' de un rol funcional. En segundo lugar, la interpretación también afirma lateralmente nuestro argumento de que las redes clientelares emergen de manea co-originaria a la diferenciación para enfrentar condiciones estructurales de alta constricción (separación de ámbitos de inclusión y exclusión) que, basados en Archer (2000, 2007), hemos sostenido limitan el modus vivendi personal. En tercer lugar, la solicitud del favor genera un compromiso de devolución, lo que reafirma la lógica transaccional temporalmente mediada de la situación social que enfatizamos en este artículo y establece el criterio normativo de cumplimiento con base en la sanción negativa de la exclusión, no solo de la red, sino también a nivel simbólico-relacional, en términos de la pérdida del estatus de 'persona'. En cuarto lugar, en referencia a las "pattern-variables" de Parsons (1970), Luhmann relaciona las redes clientelares con orientaciones de acción de tipo particularista (con foco en personas y grupos específicos) y difusa (no circunscrita a un ámbito claramente delimitado), y asocia posiciones organizacionales legalmente reguladas con criterios universalistas y específicos. Estos dos últimos puntos refieren más bien a nuestras proposiciones 3 y 4 . Esto es lo que abordamos en la sección siguiente.

3. DeFlación Y BIENES RELACIONALES: El CONTEXTO SIMBÓlico DE LA EMERGENCIA DE REDES CLIENTELARES

En esta sección nos centramos en las dos últimas proposiciones de nuestro enfoque, es decir que (3) 'las redes clientelares emergen en América Latina en una situación social de deflación de los compromisos valóricos de inclusión igualitaria y universalista, lo que promueve modalidades de acción particularizada basadas en favores que distinguen el mecanismo clientelar de transferencias mutuas' y (4) que 'las redes clientelares forman un espacio social de tipo transaccional con producción de bienes relacionales, pero con consecuencias negativas para la generalización social de esos bienes'.

Estas proposiciones hacen referencia a lo que llamamos el 'contexto simbólico' de emergencia de las redes clientelares. Al igual que en la sección anterior, buscamos sustentar teóricamente estas proposiciones y 
complementarlas con la literatura sobre América Latina y otras regiones de la sociedad mundial.

En la primera sección hemos hecho referencia a cómo las condiciones de desigualdad en América Latina conducen a un descenso continuo en la confianza institucional y a una comunitarización de los vínculos de confianza interpersonal (colegas de trabajo, vecinos, círculo familiar cercano). Esto lo observamos como un proceso de crisis de modernización, en el que la lógica sistémica del exceso afecta el modus vivendi y conduce, en condiciones sistémicas extremas, a la búsqueda de alternativas de realización de planes de vida a través de redes clientelares. Para situar teóricamente ahora el contexto simbólico de emergencia de las redes clientelares, empleamos un pasaje poco recurrido de la sociología parsoniana, el de inflación/deflación de compromisos valóricos.

Parsons (1968) sitúa este tema en el marco de la teoría general de los medios de intercambio simbólicamente generalizados. Una situación inflacionaria de los compromisos valóricos supone "sobrecompromisos": "Esto ocurre cuando una unidad ha hecho muchos compromisos, muy diversos y en tal medida serios que su capacidad para implementarlos efectivamente debe ser razonablemente puesta en duda" (Parsons 1968: 153). En América Latina, desde Germani en adelante, ha quedado claro que este es un problema central del proceso de modernización y origen de sus crisis. Los efectos de "demostración" (expectativas de estándar de vida desarrollado en sectores deprivados) y "fusión" (combinación de prácticas y actitudes modernas y tradicionales que se conciben a sí mismas como 'avanzadas') (Germani 1981: 150ss), reflejan las consecuencias del sobrecompromiso. Asimismo, en América Latina, tanto las transformaciones neoliberales (cuyo caso más radical es Chile) como los modelos populistas (cuyo paradigma hoy es Venezuela), inflacionaron a tal nivel los compromisos institucionales, que ambos países enfrentan hoy la necesidad de cambios constitucionales.

Por su parte, una situación deflacionaria consiste en la "indisponibilidad de honrar los compromisos que las unidades están dispuestas a realizar" (Parsons 1968: 153). Cuando esto acontece sostiene Parsons- se produce una fuerte limitación de la flexibilidad en la implementación de compromisos valóricos. Estos se exigen de manera más directa y mecánica, y el pluralismo valórico que caracteriza a un contexto institucional de inclusión igualitaria y universalista se transforma en alternativas dicotómicas y excluyentes que reducen los ámbitos de libertad para la consecución del modus vivendi. Las actuales antinomias 
conceptuales latinoamericanas de 'Estado o mercado', 'crecimiento o distribución', 'socialismo o muerte', 'democracia o populismo', reflejan esta situación deflacionaria de compromisos, es decir, la ausencia de pluralismo valórico. El espacio simbólico se escinde y se concentra en atractores opuestos entre sí, los que limitan la capacidad asociativa y relacional de la sociedad en diversos ámbitos y consecuentemente impiden formar una confianza social generalizada. Las redes clientelares en América Latina reproducen esta lógica deflacionaria de compromisos valóricos, en tanto oponen el esquema particular de los favores al de los derechos universales -como lo apuntamos en nuestra Proposición 4. Domingues (2008: 19) ha incluso llamado la atención sobre la generalización de la lógica clientelar en lo que denomina "clientelismo burocrático", donde el propio Estado Federal de Brasil, a través de mediadores políticos, forma comités locales de pobres para seleccionar a quienes se les entrega un beneficio monetario (Bolsa Família).

$\mathrm{Al}$ oponer el esquema de los favores al de los derechos universales, las redes se sitúan bajo una orientación de acción -como lo ha sostenido Luhmann (2005) - de tipo particularista (con foco en personas y grupos específicos) y difusa (no circunscrita a un ámbito claramente delimitado). Difícilmente esta orientación de la acción puede promover pluralismo valórico, pues "no existen amplias redes de reciprocidad potencialmente abiertas a toda la sociedad" sino solo a aquellos participantes individualizados que reproducen el esquema de favores (Hoevel 2014: 102). La consecuencia de ello es hostilidad entre grupos y desconfianza social generalizada -en otros términos, deflación de compromisos valóricos.

Adicionalmente, esta situación deflacionaria en la que se restringe el pluralismo valórico genera una fuerte presión hacia la homogenización de los modus vivendi que corre paralela a las tendencias unificadoras de una diferenciación funcional que reproduce lógicas de exceso. El espacio simbólico se reduce a alternativas 'a favor o en contra', lo que limita las posibilidades de actuar hacia el interés general y refuerza la motivación hacia la acción particularista de las redes clientelares, es decir, hacia la defensa de un modus vivendi ante las presiones por adaptarse y el riesgo de disolverse en una situación valórica dicotómica. La red clientelar refuerza entonces su compromiso particularista por medio de un esquema de favores y no de derechos universales, con lo que contribuye a la mantención de la situación deflacionaria de la que busca escapar. 
De cualquier modo, la oposición favores/derechos no es privativa de la relación entre redes clientelares y Estado latinoamericano. Según Pierre Bourdieu (2005), ella refleja el conflicto histórico constitutivo del Estado entre el principio dinástico ('la casa') y el principio jurídico (la burocracia). Este conflicto, para Bourdieu, está lejos de distinguir en forma pura a aquellos que buscan solo sus intereses individuales o de grupo de aquellos que solo buscan el bien común de modo desinteresado. Como lo ha sostenido Magdalena Tosoni (2010: 375): "los agentes obedecerán la regla de la reciprocidad o la regla de lo universal según el estado de las luchas (distribución de capitales, posiciones y beneficios resultantes) en los subcampos burocráticos". Incluso quienes siguen la regla universal, pueden buscar su propio interés y luchar por la posesión del capital económico, informacional, jurídico y simbólico, contenido dentro del capital estatal (Bourdieu 2005: 67). En este sentido, en las operaciones del Estado moderno no existe una predeterminación por la regla universal, como si ella se impusiera automáticamente por su deseabilidad (Bourdieu 2005). La orientación hacia la universalización basada en el principio jurídico o hacia el interés personal basado en el principio dinástico depende de luchas por el empleo del capital estatal y del enfrentamiento de "habitus" formados históricamente.

En tanto tienen su base en el ejercicio de un principio dinástico, las redes clientelares contribuyen a la deflación de los compromisos valóricos de inclusión igualitaria y universal. Esta situación se refuerza en la medida en que ellas permitan acceder a beneficios que de otro modo no se alcanzarían (Tosoni 2010). De este modo, el "habitus clientelar" (Rutten 2007) forma parte de las relaciones en el campo estatal, y por ello no se disuelve con simple voluntad política, tanto porque también hay voluntad política de mantenerlo, pero más básicamente porque una vez que ha emergido, logra una alta capacidad de estabilización, pues la motivación proviene de la mantención del propio modus vivendi. Una vez formado, el habitus dificulta a los agentes una acción morfogenética que trascienda la infraestructura de dominación clientelar en la que fueron entrenados. Se trata, por tanto, de una estructura social internalizada en una trama de transferencias mutuas y de relaciones intersubjetivas morfoestáticas e incluso rutinizadas por cada nueva generación.

Una alternativa teórica que puede aportar perspectivas de cambio a esta situación es la que entrega la sociología relacional (Donati 2010, 2015a; Donati \& Archer 2015). En su versión más reciente, este enfoque sostiene que "la morfogénesis de la sociedad se produce a través de 
relaciones sociales, las cuales son conectores que median entre agencia y estructura social" (Donati \& Archer 2015: 428ke). La 'relación' se entiende aquí como una distancia entre sujetos que a la vez los distingue y conecta, y que adquiere un carácter emergente, es decir, con propiedades autónomas entendidas como 'bienes' o 'males' relacionales. Lo que constituye una 'relación social' debe ser distinguido para estos autores de la conectividad y de la transacción. La primera tiene solo la forma de emisores y receptores vinculados en algún tipo de efecto de rebaño; la segunda, implica un interés objetual que sostiene la relación, la que desaparece si el interés se desvanece, es decir, no es un orden emergente con propiedades autónomas, en tanto implica "meras interacciones, intercambios, interdependencias" (Donati 2015a: 87).

Desde el inicio de este artículo hemos entendido las redes clientelares en una doble dimensión. Por un lado, ellas constituyen un sistema de transferencias mutuas, por tanto, un mecanismo de transacción. Por otro, hemos sostenido que ese sistema de transferencias se basa en un vínculo de confianza interpersonal limitado a círculos específicos, por tanto, tiene una dimensión relacional. La investigación empírica en distintas regiones de la sociedad mundial ha mostrado el carácter transaccional de las redes clientelares (Gambetta 1990, 2009; Putnam 1993, 2004; Torsello 2012; Albanese \& De Blasio 2014). Se intercambian rendimientos y accesos: favores por votos, influencias por servicios, protección por información, dinero por preferencias o privilegios. Como lo hemos sostenido en la sección 2, estas transacciones encuentran su motivación en la fuerte constricción de los modus vivendi que se produce en condiciones de crisis sistémica (Proposiciones 1 y 2). La pregunta es ahora si las redes clientelares tienen alguna posibilidad de responder a la deflación de compromisos valóricos en la cual emergen (Proposición 3), por medio de la producción de ciertos bienes relacionales (Proposición 4), aun cuando sea para su propio grupo. En otras palabras, la cuestión es si las redes clientelares "promueven una red de intercambios sociales que confiera a la relacionalidad una identidad satisfactoria sobre los agentes/actores" (Donati 2015a: 100).

Un bien relacional es un bien fundamental de personas y grupos que satisface necesidades primarias y del cual solo se puede hacer uso por medio de la relación (Donati 2015b). Para emerger requiere de identidades personales y sociales de los participantes, de una motivación no-instrumental de intercambio simbólico (no material) y que todos los participantes puedan disfrutar de él: "se trata de entidades inmateriales 
(bienes intangibles) que consisten de relaciones sociales que emergen de la reflexividad de los sujetos orientada a producir y disfrutar juntos, de manera compartida, un bien que no podrían obtener de otra manera" (Donati 2015b: 5352ke).

En las redes clientelares, estas formas de relacionabilidad alcanzan un cierto posicionamiento. Auyero (1999: 325) ha observado, para el caso argentino, cómo en las redes clientelares: "El acto de dar, las acciones de protectivas [caring] de los brokers, y la respuesta confiada de sus círculos internos transforma, o intenta transformar, una relación social contingente -la ayuda a alguien que lo necesita- en una relación reconocida (i.e. reconocida como duradera)". El mismo Auyero (2010) ha mostrado que elementos simbólicos en los discursos, como el 'amor a los pobres', la vocación de trabajadores sociales, la solidaridad con los necesitados, son una forma de eliminar la distancia burocrática y establecer relaciones de mayor socialidad, aun cuando ellas puedan luego ser la vía para poner en movimiento el mecanismo transaccional. Incluso Germani, uno de los más acérrimos críticos del peronismo argentino, reconocía en los años sesenta la oferta de un vínculo social relacional: "El dictador hizo demagogia, es verdad. Mas la parte efectiva de esa demagogia no fueron las ventajas materiales, sino el haber dado al pueblo la experiencia (ficticia o real) de que había logrado ciertos derechos y que los estaba ejerciendo" (Germani 1962: 244). Para el caso de las favelas, John Dawsey (1989) y Enrique Arias (2004) han mostrado que los vínculos de socialidad al interior de estos espacios constituyen justamente el atractivo para su cooptación por parte de agentes estatales, de agencias no-gubernamentales e incluso de carteles de droga, de manera que cuando la cooptación se produce la dimensión transaccional y la relacional se integran. Rosanne Rutten (2007), en investigaciones en Filipinas, ha incluso reconstruido la forma en que un habitus clientelar de dependencia y deferencia puede ser convertido en un habitus radical de crítica, protesta y acción colectiva, por tanto, con componentes socio-relacionales más allá de la transacción.

En nuestra perspectiva, las redes clientelares no pueden constituir bienes relacionales 'puros', en tanto su dimensión constitutiva es de tipo transaccional. Sin embargo, en condiciones sociales extremas (disparidad en acceso a sistemas, incapacidad institucional de procesamiento, separación de ámbitos de inclusión y exclusión, deflación de compromisos valóricos) ellas pueden producir sustitutos simbólicos de la confianza institucional o de una confianza interpersonal de nivel colectivo que entrega a los actores una motivación adicional (o complementaria) a la 
transacción material para mantener la red. Pueden generar un cierto nivel de vínculo recíproco, más allá de consideraciones utilitaristas (Hoevel 2013). No obstante, el problema de estos sustitutos simbólicos es que establecen un espacio normativo privatizado, fragmentado y ambiguo que coopta la autoridad pública -o es cooptado por ella- y sirve a las partes para la consecución de objetivos de corto plazo, pero en el largo plazo debilita la forma de operar de las instituciones modernas, así como sus aspiraciones de universalidad.

También en América Latina puede observarse este problema (Guasti 1977; Gordin 2002, 2006). La proliferación de redes clientelares fragmenta a las sociedades latinoamericanas en múltiples grupos cerrados en su propia lógica de intereses, con difícil comunicación con los demás y orientados hacia la búsqueda de formas de captura de las estructuras estatales para emplearlas en su propio beneficio. Esto conduce a un aparato estatal fundamentalmente corrupto (Verhezen 2005), lo que no hace sino aumentar la desconfianza en el Estado por parte de la sociedad, acentuando su fragmentación y retroalimentando constantemente todo el proceso.

\section{CONCLUSIONES}

El objetivo de este artículo ha sido perfilar teóricamente el contexto operativo y simbólico de emergencia de las redes clientelares en América Latina. Hemos situado ese contexto en el marco de una crisis de modernización en el que problemas sistémicos regulatorios ejercen fuertes presiones y limitaciones sobre los modus vivendi de las personas que no logran ser absorbidas por las instituciones sociales. Puesto que el modus vivendi contiene elementos normativos (expectativas sobre planes de vida) las personas no renuncian a él a pesar de las dificultades para realizarlo, y en tanto contiene elementos cognitivos (formas de realizar los planes de vida), las personas pueden buscar reflexivamente opciones para implementarlo (Proposición 1). Cuando las condiciones sociales son extremas (disparidades en acceso a rendimientos de sistemas sociales, incapacidad institucional de procesamiento de demandas, separación de ámbitos de inclusión y exclusión, desconfianza institucional), las redes clientelares constituyen una alternativa supletoria para sostener los planes de vida ante la precariedad de las respuestas institucionales y sus consecuencias homogeneizantes (Proposición 2). No obstante, el marco simbólico en el que emergen es el de una deflación de compromisos 
valóricos de inclusión igualitaria y universalista producida por la situación de crisis. En esta situación deflacionaria, el pluralismo valórico se reduce drásticamente. La incorporación de ciertos grupos en redes clientelares puede ser motivada por la mantención de un horizonte valórico propio, sin embargo, puesto que estas redes oponen el principio de los favores al de los derechos universales, reproducen esta lógica deflacionaria de la que intentan escapar (Proposición 3).

A pesar de su carácter fundamentalmente transaccional como sistema de transferencias mutuas, las redes clientelares pueden aportar determinados bienes relacionales que hacen frente a la deflación de compromisos valóricos. Sin embargo, en tanto se trata de ámbitos de confianza limitada y excluyente, ellas establecen un espacio normativo fragmentado y ambiguo en el que las condiciones de confianza que pueden producir están impedidas de capacidad de generalización y de la posibilidad de una recomposición amplia de la decreciente confianza social institucional que tiene lugar en el marco de la crisis de modernización (Proposición 4).

Para esta argumentación hemos recurrido sostenidamente a la teoría sociológica general contemporánea y a distintas investigaciones empíricas realizadas preferentemente en América Latina. Más que optar por un modelo teórico determinado, hemos preferido centrarnos en el 'objeto redes clientelares' y a partir de ahí explorar alternativas de apoyo teórico, complementariedades y relaciones conceptuales. El objetivo de esta estrategia metodológica ha sido expandir el espectro de resonancia teórica y las posibilidades de interpretación de este fenómeno tan ampliamente estudiado en América Latina.

A partir de estos objetivos (el primero teórico, el segundo metodológico), identificamos dos limitaciones de nuestra investigación, una relativa a la dimensión teórica, otra relativa a la dimensión empírica. En el plano teórico, no hemos explorado la importante contribución que pueden realizar a esclarecer aspectos del fenómeno de las redes clientelares en América Latina teorías como el rational choice (Coleman, Becker), el enfoque post-estructuralista (Deleuze \& Guattari), o recientes teorías sociológicas de redes (White, Latour). Una razón de esto es la extensión del texto, pero más sustantivamente el fundamento es que hemos seleccionado teorías sociológicas generales en las que las redes no son un objeto específico, pero que abordan el tema como una derivación de sus arquitecturas conceptuales $\mathrm{y}$, en tal medida, las integran en un marco analítico de amplio alcance. 
En el plano empírico, la investigación acerca de los componentes simbólicos y relacionales (en el sentido de Donati y Archer) no es abundante en América Latina. Auyero ha puesto sistemáticamente el acento en la necesidad de investigación no solo de la dimensión transaccional de las redes clientelares o en su aspecto de dominación, sino también en la perspectiva de sus consecuencias simbólicas para los involucrados. En buena parte, su propia investigación ha apuntado a llenar este vacío, pero se requiere aún una exploración más profunda, teóricamente guiada, de los modos en que las redes clientelares producen morfogenética y morfoestáticamente compromisos valóricos, y cómo generan bienes relacionales de alcance limitado. Si bien desde Marcel Mauss en adelante la investigación antropológica es abundante en análisis de sistemas de reciprocidad de tipo tradicional, la alta interpenetración actual de las redes clientelares en ámbitos modernos y en contextos de fuerte globalización requiere de nuevas investigaciones empíricas en este campo.

En este sentido, las líneas de investigación que se abren desde nuestras reflexiones apuntan a observar aspectos distintos del contexto operativo y simbólico de emergencia de las redes clientelares en América Latina para los que las teorías sociológicas generales no empleadas en este artículo puedan aportar elementos interpretativos y explicativos. Del mismo modo, la investigación empírica en los aspectos relacionales de las redes clientelares en América Latina, específicamente la producción de 'bienes' y 'males' relacionales en la línea de Donati y Archer, puede ser un campo fértil para poner a prueba proposiciones teóricas recientes $\mathrm{y}$, a la vez, aportar al estudio de los componentes simbólicos de aquellas. Esto puede arrojar pistas sobre cómo combinar la lógica funcional estatal, mercantil o jurídica con la dimensión civil-relacional para producir, de un modo universal y legítimo, la capilaridad social que las redes clientelares alimentan de modo negativo y cuya dinámica vemos también resurgir en distintas partes del mundo.RM

\section{REFERENCIAS}

Aguirre, J. (2012). Redes Clientelares. Una perspectiva teórica desde el análisis de redes sociales. Documentos de Trabajo 83, Centro Interdisciplinario para el Estudio de Políticas Públicas. Buenos Aires: Ciepp.

Albanese, G. \& de Blasio, G. (2014). Who Trust Other More? A Cross-European Study. Empirica, 41, 803-820.

Araujo, K. (2009). Habitar lo social. Santiago: Lom Ediciones.

Archer, M. (2000). Being Human. Cambridge: Cambridge University Press. 
Archer, M. (2007). Making our Way Through the World. Cambridge: Cambridge University Press.

Archer, M. (2009). Teoría social realista. El enfoque morfogenético. Santiago: Ediciones Universidad Alberto Hurtado.

Archer, M. (Ed.) (2013). Social Morphogenesis. Dordrecht: Springer.

Arias, E. (2004). Faith in Our Neighbors: Networks and Social Order in Three Brazilian Favelas. Latin American Politics and Society, 46(1), 1-38.

Arias, E. \& Rodrigues, C. (2006). The Myth of Personal Security: Criminal Gangs, Dispute Resolution, and Identity in Rio de Janeiro's Favelas. Latin American Politics \& Society, 48(4), 53-81.

Auyero, J. (1999). 'From the Client's Point(s) of View': How Poor People Perceive and Evaluate Political Clientelism. Theory and Society, 28, 297-334.

Auyero, J. (2000). The Logic of Clientelism in Latin America: An Ethnographic Approach. Latin American Research Review, 35(3), 55-81.

Auyero, J. (2010). Visible Fists, Clandestine Kicks, and Invisible Elbows: Three Forms of Regulating Neoliberal Poverty. European Review of Latin American and Caribbean Studies, 89, 5-26.

Auyero, J. (2012). Poor People's Lives and Politics: The Things a Political Ethnographer Knows (and doesn't Know) After 15 Years of Fieldwork. New Perspectives on Turkey, 46, 95-127.

Bourdieu, P. (2005). De la casa del rey a la razón de Estado. Un modelo de la génesis del campo burocrático. En L. Wacquant (coord.), El misterio del ministerio. Pierre Bourdieu y la política democrática (pp. 43-70). Barcelona: Gedisa.

Bringel, B. y Domingues, J. (2015). Global Modernity and Social Contestation. London, SAGE.

Cadenas, H. (2012a). La desigualdad de la sociedad. Diferenciación y desigualdad en la sociedad moderna. Persona y Sociedad, 26(2), 51-77.

Cadenas, H. (2012b). Algunas tendencias de la diferenciación del derecho en Chile. Século XXI - Revista de Ciências Sociais, 2(1), 72-107.

Cadenas, H. (2016). Desigualdad social y teoría de sistemas: la importancia de los medios. Economía y Política, 3(1), 41-69.

Callon, M. (1998). An Essay on Framing and Overflowing: Economic Externalities Revisited by Sociology. The Sociological Review, 46, 244-269

CEPAL (2014). Panorama social de América Latina. Santiago: CEPAL.

CEPAL (2015). Desarrollo social inclusivo. Santiago: CEPAL.

Chávez, J. \& Mujica, F. (2015). Sociología de la cooptación: la imposibilidad de ser elite en América Latina y la reforma educacional chilena como testimonio de la ideología de la modernidad inducida. Persona y Sociedad, 29(1), 63-84.

Chernilo, D. (2007). A Social Theory of the Nation-State. London: Routledge.

Cousiño, C. (1990). Razón y ofrenda. Santiago: Universidad Católica de Chile.

Dawsey, J. (1989). Organização do espaço em favelas: Disciplina e reciprocidade. Impulso, 3(6), 7-16.

Deffner, V. (2007). Soziale Verwundbarkeit im 'Risikoraum Favela' — Eine Analyse des sozialen Raumes auf der Grundlage von Bourdieus 'Theorie der Praxis'. En R. Wehrhahn (ed.), Risiko und Vulnerabilität in Lateinamerika (pp. 207-232). Kiel: Kieler Geographische Schriften 117. 
Domingues, J. (2008). Latin America and Contemporary Modernity: A Sociological Interpretation. London: Routledge.

Donati, P. (2011). Relational Sociology. London: Routledge.

Donati, P. (2015a). Manifesto for a Critical Realist Relational Sociology. International Review of Sociology, 25(1), 86-109.

Donati, P. (2015b). When Relational Subjects Generate Relational Goods. En P. Donati \& M. Archer, The Relational Subject (pp. 198-228). Cambridge: Cambridge University Press (Kindle Edition).

Donati, P. \& Archer, M. (2015). The Relational Subject. Cambridge: Cambridge University Press (Kindle Edition).

Durston, J. (1999). Construyendo capital social comunitario. Revista de la CEPAL, 69, 103-118.

Eisenstadt, S. \& Roniger, L. (1984). Patrons, Clients and Friends: Interpersonal Relations and the Structure of Trust in Society. Cambridge: Cambridge University Press.

Escobar, C. (2002). Clientelism and Citizenship: The Limits of Democratic Reform in Sucre, Colombia. Latin American Perspectives, 29(5), 20-47.

Feltran, G. (2010). Crime e castigo na cidade: os repertórios da justiça e a questão do homicídio nas periferias de São Paulo. Caderno CRH, 23(58), 59-73.

Gambetta, D. (1990). Mafia: The Price of Distrust. En D. Gambetta (Ed.), Trust: Making and Breaking Cooperative Relations (pp. 158-175). Oxford: Oxford University Press.

Gambetta, D. (2009). Codes of the Underworld. How Criminals Communicate. Princeton: Princeton University Press.

Garretón, M. (2000). La sociedad en que vivi(re)mos. Santiago: LOM.

Germani, G. (1962). Política y sociedad en una época en transición. Buenos Aires: Paidós.

Germani, G. (1981). The Sociology of Modernization. London: Transaction Books.

Girola, L. (2011). La cultura de la transgresión. Anomias y cultura del 'como si' en la sociedad mexicana. Estudios Sociológicos, 29(85), 99-129.

Gordin, J. (2002). The Political and Partisan Determinants of Patronage in Latin America, 1960-1994: A Comparative Perspective. European Journal of Political Research, 41, 513-549.

Gordin, J. (2006). La sustentabilidad política del clientelismo: teoría y observaciones empíricas en América Latina. Documentos CIDOB 13. Barcelona: CIDOB.

Guasti, L. (1977). Peru: Clientelism and Internal Control. En Schmidt, S. (Ed.), Friends, Followers, and Factions: A Reader in Political Clientelism (pp. 422-438). Berkeley: University of California Press.

Habermas, J. (1973). Legitimation Crisis. Cambridge: Polity Press.

Haldane, A. \& May, R. (2011). Systemic Risk in Banking Ecosystems. Nature, 469, 351355.

Heinrichs, D., Hansjürgens, B., \& Krellenberg, K. (Eds.) (2011). Risk Habitat Megacity. Berlin: Springer Verlag.

Helmke, G. \& Levitsky, S. (Eds.) (2006). Informal Institutions and Democracy. Baltimore: The Johns Hopkins University Press.

Hoevel, C. (2013). The Economy of Recognition. Dordrecht: Springer.

Hoevel, C. (2014). La economía del don: modelos y realidades. En O. Groppa \& C. Hoevel (Eds.), Economía del don. Perspectivas para Latinoamérica (pp. 85-118). Ciudad Nueva: Buenos Aires. 
Holzer, B. (2006). Netzwerke. Bielefeld: transcript Verlag.

Huguet, C. \& De Carvalho, I. (2008). Violence in the Brazilian Favelas and the Role of the Police. New Directions in Youth Development, 119, 93-109.

Kitschelt, H. \& Wilkinson, S. (2007). Patrons, Clients and Policies: Patterns of Democratic Accountability and Political Competition. Cambridge: Cambridge University Press

Larraín, J. (2000). Identity and modernity in Latin America. Cambridge: Polity Press.

Latinobarómetro (2015). La confianza en América Latina. Santiago: Latinobarómetro.

Lechner, N. (1999). El Estado en el contexto de la modernidad. En R. Lechner, R. Millán, \& F. Valdés (Eds.), Reforma del Estado y coordinación social (pp. 39-54) México: Plaza y Valdés.

Lomnitz, L. (1988). Informal Exchange Networks in Formal Systems: A Theoretical Model. American Anthropologist, 90(1), 42-55.

Luhmann, N. (1997). Globalization or World Society: How to Conceive of Modern Society? International Review of Sociology, 7(1), 67-79.

Luhmann, N. (2000). Vertrauen. Stuttgart: Lucius \& Lucius Verlagsgesellschaft.

Luhmann, N. (2005). Inklusion und Exklusion. En Soziologische Aufklärung 6 (pp. 226251). Wiesbaden: VS Verlag.

Luhmann, N. (2007). La sociedad de la sociedad. México: Herder.

Mascareño, A. (2012). Die Moderne Lateinamerikas. Bielefeld: transcript Verlag.

Mascareño, A., Goles, E., \& Ruz, G. (2016). Crisis in Complex Social Systems: A Social Theory View Illustrated with the Chilean Case. Complexity, DOI 10.1002/cplx.21778.

Maturana, H. (2009). La realidad: ¿Objetiva o construida? Barcelona: Anthropos.

Mignolo, W. (2005). La idea de América Latina. Barcelona: Gedisa.

Morandé, P. (1987). Cultura y modernización en América Latina. Madrid: Ediciones Encuentro.

Narotzky, S. \& Moreno, P. (2002). Reciprocity's Dark Side. Negative Reciprocity, Morality and Social Reproduction. Anthropological Theory, 2(3), 281-305.

Neves, M. (2007). A constitutionalização simbólica. São Paulo: Editora Acadêmica.

Oliveros, V. (2013). A Working Machine. Patronage Jobs and Political Services in Argentina. Doctoral Thesis Columbia University. Online en: http://academiccommons.columbia.edu/catalog/ac:161503 [2 de enero 2016].

Parker, C. (1996). Otra lógica en América Latina. México: Fondo de Cultura Económica.

Parsons, T. (1968). On the Concept of Value Commitments. Sociological Inquiry, 38(2), $135-160$.

Parsons, T. (1970). The Social System. London: Routledge \& Kegan Paul.

Poggi, G. (1978). The Development of Modern State. Stanford, CA: Stanford University Press.

Putnam, R. (1993). Making Democracy Work: Civic Traditions in Modern Italy. Princeton: Princeton University Press.

Putnam, R. (Ed.) (2004). Democracies in Flux. Oxford: Oxford University Press.

Rothstein, B. \& Stolle, D. (2003). Introduction: Social Capital in Scandinavia. Scandinavian Political Studies, 26(1), 1-26.

Rutten, R. (2007). Losing Face in Philippine Labor Confrontations: How Shame May Inhibit Worker Activism. En L. Joseph, M. Mahler, \& J. Auyero (Eds.), New Perspectives in Political Ethnography. New York: Springer. 
Stichweh, R. (2005). Inklusion und Exklusion. Studien zur Gesellschaftstheorie. Bielefeld: transcript Verlag.

Teubner, G. (2012). Constitutional Fragments. Oxford: Oxford University Press.

Torsello, D. (2012). Clientelism and Social Trust in Comparative Perspective: Particularism versus Universalism. International Journal of Humanities and Social Science, 2(23), 71-78.

Tosoni, M. (2010). Niklas Luhmann y Pierre Bourdieu: claves teóricas para la interpretación del clientelismo político en la Argentina. Estudios Sociológicos, 28(83), 359-381.

Verhezen, P. (2005). Gifts and Bribes. An Essay on the Limits of Reciprocity. Leuven: Hoger Instituut Voor Wijsbegeerte.

Willke, H. (2014). Concepciones de regulación política y problemas de gobierno político. Economía y Política, 1(1), 79-118.

\section{SOBRE LOS AUTORES}

Carlos Hoevel es Doctor en Filosofía (Pontificia Universidad Católica Argentina) y Master of Arts in the Social Sciences (University of Chicago). Profesor de Historia de las Ideas Económicas y Políticas, Filosofía Social y Filosofía de la Economía en el Departamento de Economía de la Pontificia Universidad Católica Argentina. Es también Director del Centro de Estudios en Economía y Cultura y de la Revista Cultura Económica de la misma Universidad. Sus áreas de investigación incluyen filosofía social, filosofía de la economía y teoría social. Algunas publicaciones recientes: The Economy of Recognition (Springer, Heidelberg-New York, 2013), La economía del don. Perspectivas para América Latina, (Octavio Groppa \& Carlos Hoevel eds., Ciudad Nueva, Buenos Aires 2014) y Rosmini: A Philosopher in Search of the Economy, Journal of History of Economic Thought and Policy, 2014, Vol. 2.

Aldo Mascareño es Doctor en Sociología (Universidad de Bielefeld, Alemania). Profesor de Sociología de la Escuela de Gobierno de la Universidad Adolfo Ibáñez, Chile, y Director del Doctorado en Procesos e Instituciones Políticas de la misma universidad. Es también Investigador Principal del Núcleo Milenio Modelos de Crisis (Iniciativa Científica Milenio). Actualmente sus intereses de investigación incluyen teoría sociológica contemporánea, sociología de América Latina y teorías de crisis. Es autor de Die Moderne Lateinamerikas (transcript Verlag 2012), Diferenciación y contingencia en América Latina (Ediciones UAH 2010), y coeditor of Durch Luhmanns Brille (Springer Verlag 2012), y Legitimization in World Society (Ashgate Publishing 2012). El autor agradece al Núcleo Milenio Modelos de Crisis (NS 130017) por el apoyo en esta investigación.

\section{CONTACTO}

CARLOS HoEvel

Centro de Estudios en Economía y Cultura

Facultad de Ciencias Económicas 
Pontifica Universidad Católica Argentina

Avenida Alicia Moreau de Justo 1300

Buenos Aires, Argentina

CP: 1107AAZ

carlos_hoevel@uca.edu.ar

Aldo MASCAREÑO

Escuela de Gobierno

Universidad Adolfo Ibáñez

Diagonal Las Torres 2640

Santiago, Chile

CP: 7941169

aldo.mascareno@uai.cl

Recibido: Diciembre 2015

Aceptado: Abril 2016 